\title{
The RELATION OF SERUM IRISIN LEVEL WITH METABOLIC AND HORMONAL CHANGES IN RAT MODEL OF POLYCYSTIC OVARY
}

\author{
Mohamed Hussein Ibrahim, Soad Abd Allah Ahmed, Doaa Attiaa Abdel Moety, Sherein Fathy \\ Elsayed \\ Physiology Department, faculty of medicine, Zagazig University \\ Corresponding author: Sherein Fathy, dr_sfathy@hotmail.com
}

\section{ABSTRACT}

Background: Polycystic ovary syndrome (PCOS) is one of the leading causes of infertility in female. It is usually associated with insulin resistance (IR), obesity, hyperlipidemia, and type II diabetes. Irisin is amyokine which increases energy expenditure and protects against insulin resistance and obesity. There were controversial studies about the levels of irisin and its relation to hormonal and metabolic changes in PCOS. This study was designed to estimate serum level of irisin in letrozole-induced PCOS in both lean and obese female rats, and to study the link between serum irisin level and some metabolic and hormonal parameters in PCOS.

Material and Methods: This study was conducted on 48 young virgin female of local strain albino rats. They were randomly divided into 2 equal groups: Group I: lean group were fed on normal laboratory chow diet and Group II: A high fat diet induced obesity group and each group was subdivided into group A (control) and group B (letrozole induced PCOS). Fasting serum irisin, Lutenizig hormone (LH), Folliclular stimulating hormone (FSH), testosterone, estradiol, progesterone, insulin, glucose, triglycerides, cholesterol, LDL and HDL levels were estimated. In addition, body mass index (BMI) and Homeostatic Model Assessment of Insulin Resistance (HOMA-IR) were calculated in all groups at the end of the experiment.

Results: Serum irisin level was elevated in both lean and obese PCOS groups when compared to both lean and obese control groups. In addition, obese PCOS group had significant higher level of irisin when compared to lean PCOS group.

Conclusion: Serum irisin level was positively correlated with BMI, HDL and testosterone levels and negatively correlated with HOMA- IR, insulin, cholesterol, triglycerides and LDL levels. Irisin may have a role in the development of polycystic ovary syndrome.

Key words: Irisin - PCOS- lean - obese.

\section{INTRODUCTION}

$\mathrm{I}^{\mathrm{r}}$ risin is a newly discovered exercisemediated myokine secreted by skeletal muscle and play an important role in regulation of energy metabolism by inducing browning of white adipose tissue ${ }^{[1]}$. Irisin is also released by adipocytes ${ }^{[2]}$. The regulation of irisin as well as its role in glucose metabolism remains to be clarified ${ }^{[2,3]}$.

Irisin is also related to increased incidence of metabolic syndrome and cardiovascular disease in humans, and its secretion may be increased as a compensatory mechanism to overcome an underlying irisin resistance in these subjects ${ }^{[4]}$.

PCOS is a hormonal disorder common among women of reproductive age. ${ }^{[5]}$. It is characterized by hyperandrogenism, ovulatory dysfunction and infertility ${ }^{[6]}$.

Also, it is associated with insulin resistance, obesity, hyperlipidemia, increased prevalence of type II diabetes, endothelial dysfunction and oxidative stress ${ }^{[7,8]}$.
Few researchers illustrate the relationship between serum irisin level and PCOS patients ${ }^{[\mathbf{9}, \mathbf{1 0}]}$. So, this present study tries to elucidate this relationship, by estimating the level of serum irisin in lean and obese PCOS rats and also, by searching for the link between serum irisin level and BMI, IR, and lipid profile in both lean and obese PCOS rats.

\section{MATERIAL AND METHODS}

This study was carried out on 48 young virgin female of local strain albino rats of 6 weeks old weighing 90-100 gm. They were obtained from the animal house faculty of veterinary medicine Zagazig University.

The rats were kept in steel wire cages (6/cages) in the animal house of the faculty of medicine Zagazig University under hygienic conditions.

The rats had free access to water and chow, were kept at room temperature and were maintained on a $12 \mathrm{~h}$ light/ dark cycle. The experimental protocol was approved by physiology department and by local medical 
ethics committee in faculty of medicine of Zagazig University (Institutional Review Board, IRB).

The animals were randomly divided into 2 main groups $(n=24)$ : Group I : lean rats, fed on commercial rat standard chow consisted of $25.8 \%$ protein, $62.8 \%$ carbohydrates and $11.4 \%$ fat Group II: high fat diet induced obese rats "HFD" rats fed on high fat diet generally contain protein $20 \%$, carbohydrates $35 \%$ and fat $45 \%$, mainly in form of lard and soy bean for 9 weeks ${ }^{[11]}$. Then each group further subdivided into subgroup A (control) given $1 \mathrm{ml}$ water orally by gavage daily for 21 days, subgroup B (letrozole induced PCOS): oral administration of letrozole (non-steroidal aromatase inhibitor, ACDIMA international) (Daily 0.5 $\mathrm{mg} / \mathrm{kg}$ dissolved in water by gavage) for 21 consecutive day ${ }^{[12]}$.

Determination of Sexual cycle: Smears were obtained daily by vaginal washing with saline. The fresh unstained samples were evaluated microscopically during the treatment period ${ }^{[12]}$. Cycles with duration of 4 to 5 days were considered regular estrus phases were determined according to Marcondes et al. [13] and Goldman et al. ${ }^{[14]}$ by examining the vaginal smear into:

- The proestrus phase: the vaginal smear consists of a predominance of nucleated epithelial cells with smooth margins.

-The estrus phase: the vaginal smear shows large anucleated cornified (keratinized) cells with irregular margins.

-The met estrus phase: the vaginal smear shows many cornified cells plus infiltration of leukocytes.

- The diestrus phase: the vaginal smear shows absence of the cornified cells and presence of small leukocytes.

The observation of cornified cells in the smears during a minimum of 10 consecutive days was defined as persistent estrous, indicating anovulation and development of follicular cysts.

Calculation of BMI: $24 \mathrm{~h}$ after the end of the study (after the last dose of letrozole), and after overnight fasting, rats weighed and BMI were calculated according to Novelli et al. ${ }^{[15]}$ from the following equation:

$\mathrm{BMI}=\underline{\text { body weight }(\mathrm{gm})}$ length ${ }^{2}\left(\mathrm{~cm}^{2}\right)$ (nose to anus)

Collection of blood and tissue samples: Rats were anaesthetized by ether inhalation, blood samples were collected from orbital sinus (sampling of controls taken in the estrus phase) and ovaries were dissected and immediately fixed in $4 \%$ paraformaldehyde. Blood centrifuged at $3000 \mathrm{rpm}$ for 15 minutes, the supernatant serum was stored at $20^{\circ} \mathrm{C}^{[16]}$ until used for:

Estimation of serum irisin level, according to Bostram et al. ${ }^{[1]}$ using rat irisin ELISA rat kit (Catalog \# K4761-100), biovision, Milpitas Blvd., Milpitas, CA 95035 USA was performed. Estimation of serum LH, FSH, estradiol, progesterone and testosterone levels: according to Tietz ${ }^{[17]}$.using rat kits: BC-1031, BC-1029, BC-1111, BC-1113 and BC-1115, respectively, BioCheckInc 323 Vintage Park Dr. Foster City, CA - 94404.

Estimation of serum glucose level according to Tietz ${ }^{[17]}$ and serum insulin level by enzyme- linked immunosorbent assay (ELISA) according to Temple et al. ${ }^{[18]}$. Kits for estimation of serum glucose and insulin levels were purchased from (Biosource Europe S.A.Belgium).

Measurement of homeostasis model assessment (HOMA-IR) index was calculated according to Sun et al. ${ }^{[19]}$ as follows:

[HOMA-IR] $=$ fasting serum glucose $(\mathrm{mg} / \mathrm{dl})$ $\times$ fasting serum insulin $(\mu \mathrm{IU} / \mathrm{ml}) / 405$.

Estimation of lipid profile as follows: Total serum cholesterol level: according to Tietz ${ }^{[17]}$ [serum TG level: according to Fossati ${ }^{[20]}$, serum HDL levels according to Nauck et al. [21] and serum LDL levels was calculated according to Friedewald et al. ${ }^{[22]}$ as follows: $\mathrm{LDL}=\mathrm{TC}-\mathrm{HDL}-\mathrm{TG} / 5$ (Kits for estimation of serum glucose, insulin, cholesterol, TG and HDL levels were purchased from Biosource Europe S.A.Belgium).

Histopathological examination: The abdominal cavities of the rats were opened. Ovaries were dissected and fixed in $10 \%$ buffered formalin for 6 hours at room temperature and washed in a phosphate buffer saline solution. For light microscopy, fixed tissues were dehydrated in an ascending series of ethanol, cleared in xylene and embedded in paraffin. $5 \mathrm{~mm}$ thick sections were mounted in slides previously treated with 3- 
aminopyropyl triethoxysilane and stained with hematoxylin-eoisin preliminary observation ${ }^{[23]}$. The pathologist was blinded to the treatment.

Statistical analysis: The data obtained in the present study were expressed as mean \pm SD for quantitative variables and statistically analyzed according to the methods described by Kirkwood ${ }^{[24]}$. The statistical analysis is done by using SPSS program (19) (SPSS Inc. Chicago, IL, USA).

ANOVA (Post hoc) test was used to compare means among more than two groups.

$\mathrm{P}$ value $<0.05$ was considered statistically significant.

Corrélation coefficient $(r)$ :Pearson's correlation analysis was performed to illustrate the relationships between serum irisin and the studied metabolic parameters among different groups. Pearson's correlation was considered significant at $P$ values $<0.05$.

\section{Histopathological findings \\ RESULTS}

Ovaries from the control groups (IA and IIA groups) histologically (under 200_ magnification) had numerous variable sized Graffian follicles at different stages of maturation surrounded by dense spindle shaped ovarian stroma (figure 1 \&2).

In ovaries from PCO rats (IB and IIB groups) histologically (under 200 magnification), it showed numerous cystically dilated follicles surrounded by dense ovarian stroma (Figure 3 \& 4).

This study revealed that in lean PCOS group (IB) there were significant high levels of serum irisin, $\mathrm{LH}$, testosterone and $\mathrm{BMI}$ when compared with that of lean control group (IA), (P value: < $0.001,<0.001,<0.001,<\quad 0.05$ respectively). While there were significant low levels of serum estradiol and progesterone in group (IB) compared to control group (P value: < $0.001,<0.001$ respectively), however, no significant change in serum $\mathrm{FSH}$, insulin, glucose, calculated HOMA, serum cholesterol, triglycerides, LDL and HDL levels, ( $\mathrm{P}$ value: > 0.05).

Moreover, in obese PCOS group (IIB) there were significant high levels of serum irisin, LH, testosterone, insulin, cholesterol, triglycerides , LDL levels, BMI and calculated HOMA-IR compared with that of obese control Group (IIA), (P value: $<0.001,<0.001,<0.001,<0.001,<$ $0.001,<0.001,<0.001,<0.001,<0.001$

respectively). While there were significant low levels of serum estradiol ,progesterone and HDL compared with that of obese control,(P value: $<0.001,<0.001,<0.001$ respectively), however, no significant change in serum FSH and glucose, $(\mathrm{P}>0.05)$.

Also, obese group (IIA) showed significant high levels of serum irisin, glucose, insulin, cholesterol, triglycerides, LDL levels, BMI and calculated HOMA-IR compared with that of lean control (IA) (P value: $<0.001,<0.001,<0.001<0.001,<$ $0.001,<0.001,<0.001,<0.001$ respectively and significant low levels of serum HDL (P value: < 0.001). However there were no significant change in serum levels of LH, $\mathrm{FSH}$, testosterone, estradiol and progesterone between the two groups $(\mathrm{P}>0.05)$.

In addition, obese PCOS group (IIB) showed significant high levels serum irisin, LH, glucose, insulin, cholesterol, triglycerides, LDL levels, BMI and calculated HOMA-IR compared with that of lean control (IB) $\quad(\mathrm{P}$ value: $<0.001,<0.05$, $<0.001,<0.001,<\quad 0.001,<\quad 0.001,<$ $0.001,<0.001$, respectively) and significant low levels of serum HDL (P value: < 0.001). However there were no significant change in serum levels of FSH, testosterone, estradiol and progesterone between the two groups ( $\mathrm{P}$ $>0.05$ ) (Table 1; Figures 5-7).

Irisin showed significant positive correlation with BMI, testosterone and HDL levels in (Group IB: $\mathrm{r}=0.779 * * \mathrm{p}<0.01, \mathrm{r}=$ $0.921 * * * \mathrm{p}<0.001, \mathrm{r}=0.879 * * * \mathrm{p}<0.001$ respectively),(Group IIA: $\mathrm{r}=0.753 * * \mathrm{p}<0.01$, $\mathrm{r}=0.879 * * * \mathrm{p}<0.001, \mathrm{r}=0.781 * * \mathrm{p}<0.01$ respectively), (Group IIB: $r=0.779^{* *} \mathrm{p}$ $<0.01, \mathrm{r}=0.921^{* * * *} \mathrm{p}<0.001, \mathrm{r}=0.867^{* * *} \mathrm{p}$ $<0.001$ respectively), while irisin showed significant negative correlation with insulin, HOMA IR, cholesterol, triglycerides and LDL levels in (Group IB: $\mathrm{r}=-0.885^{* * *} \mathrm{p}<0.001$, $\mathrm{r}=-0.912 * * * \mathrm{p}<0.001, \mathrm{r}=-0.724 * * * \mathrm{p}<0.01$, $\mathrm{r}=-0.905 * * * \mathrm{p}<0.001, \mathrm{r}=-0.902 * * * \mathrm{p}<0.001$ respectively), (Group IIA: $\mathrm{r}=-0.871 * * * \mathrm{p}$ $<0.001, \mathrm{r}=-0.888 * * * \mathrm{p}<0.001, \mathrm{r}=-0.754 * * \mathrm{p}$ $<0.01, r=-0.776^{* *} \mathrm{p}<0.01, \mathrm{r}=-0.680 * \mathrm{p}$ 
$<0.05$ respectively), (Group IIB: $r=-0.885^{* * *}$ $\mathrm{p}<0.001, \mathrm{r}=-0.818^{* * *} \mathrm{p}<0.001, \mathrm{r}=-0.724^{* *} \mathrm{p}$ $<0.01, \quad r=-0.905^{* * *} \mathrm{p}<0.001, \quad r=-0.902^{* * *} \mathrm{p}$ $<0.001$ respectively). However, no

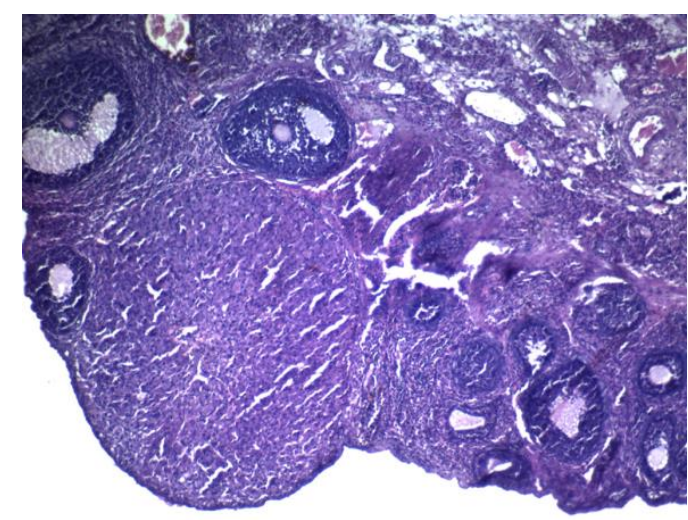

Figure 1: Histologic ovarian tissue sections dyed with $\mathrm{H} \& \mathrm{E}$ in group IA (lean control) under 200_ magnification.

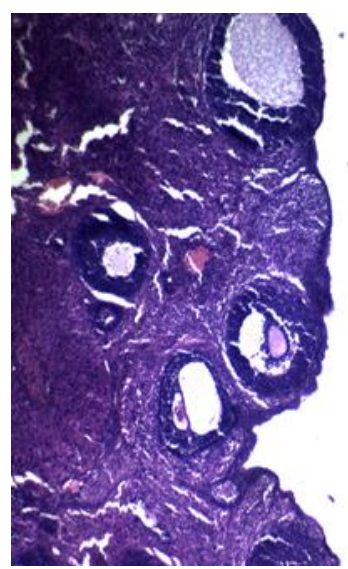

Figure 3: Histologic ovarian tissue sections dyed with $\mathrm{H} \& \mathrm{E}$ in group IIA (obese control) under 200_ magnification. significant correlation between irisin, LH, FSH, estradiol, progesterone and glucose levels in those groups $(\mathrm{P}>0.05)$.

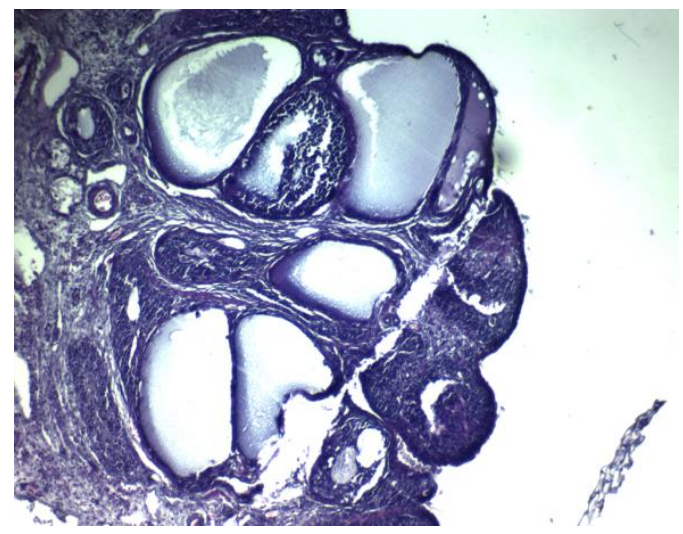

Figure 2: Histologic ovarian tissue sections dyed with H\&E in group IB (lean PCOS) under 200_ magnification.

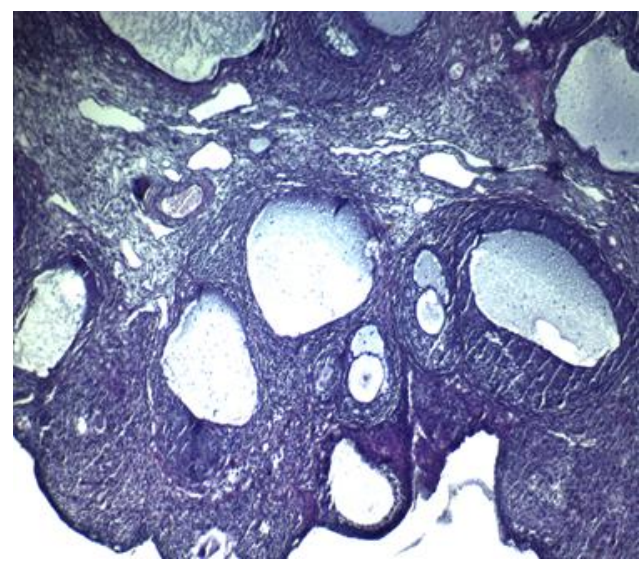

Figure 4: Histologic ovarian tissue sections dyed with H\&E in group IIB (obese PCOS) under 100 magnification. 
Table 1: Shows all measured parameters in all studied groups expressed as (mean \pm SD):

\begin{tabular}{|c|c|c|c|c|}
\hline $\begin{array}{c}\text { groups } \\
\text { parameters } \\
\end{array}$ & Group IA & Group IB & Group IIA & Group IIB \\
\hline irisin(nglml) & $7.57 \pm 1$ & $11.16 \pm 0.98^{* * * a}$ & $15.53 \pm 1.3^{* * * a, b}$ & $17.7 \pm 1.5^{* * * a, b, c}$ \\
\hline BMI (gm/cm2) & $0.48 \pm 0.028$ & $0.53 \pm 0.031^{* a}$ & $0.83 \pm 0.038^{* * * a, b}$ & $0.89 \pm 0.052^{* * * a, b, c}$ \\
\hline LH (IU/ml) & $2.16 \pm 0.29$ & $5.96 \pm 0.76^{* * * a}$ & $2.31 \pm 0.29$ & $6.5 \pm 0.84^{* * * a, c, * b}$ \\
\hline FSH (IU/ml) & $3.76 \pm 0.31$ & $3.82 \pm 0.34$ & $3.76 \pm 0.32$ & $3.98 \pm 0.22$ \\
\hline estradiol $(\mathrm{pg} / \mathrm{ml})$ & $31.4 \pm 4.26$ & $14.2 \pm 2.18^{* * * a}$ & $32.03 \pm 4.2$ & $13.69 \pm 2.3^{* * * a, c}$ \\
\hline $\begin{array}{l}\text { Progesterone } \\
(\mathrm{pg} / \mathrm{ml})\end{array}$ & $7.7 \pm 0.73$ & $5.59 \pm 0.96^{* * * a}$ & $7.81 \pm 0.97$ & $5.02 \pm 0.99^{* * *_{\mathrm{a}, \mathrm{c}}}$ \\
\hline $\begin{array}{l}\text { Testosterone } \\
(\mathrm{pg} / \mathrm{ml})\end{array}$ & $77.75 \pm 10.4$ & $233.7 \pm 12.6^{* * * a}$ & $80.6 \pm 4.5$ & $235.3 \pm 12.5^{* * * a, c}$ \\
\hline Insulin $(\mu \mathrm{IU} / \mathrm{ml})$ & $12.35 \pm 1.55$ & $13.29 \pm 1.45$ & $22.5 \pm 1.2^{* * * a, b}$ & $26.25 \pm 2.23^{* * * a, b, c}$ \\
\hline Glucose (mg/ dl) & $84.6 \pm 6.2$ & $85 \pm 5.6$ & $140.8 \pm 6.3^{* * * a, b}$ & $143.75 \pm 4.3^{* * * a, b}$ \\
\hline HOMA-IR & $2.58 \pm 0.39$ & $2.8 \pm 0.44$ & $7.83 \pm 0.61^{* * * a, b}$ & $9.33 \pm 0.98^{* * * a, b, c}$ \\
\hline $\begin{array}{l}\text { total cholesterol } \\
(\mathrm{mg} / \mathrm{dl})\end{array}$ & $162.5 \pm 16.16$ & $166.3 \pm 15.7$ & $235 \pm 16.5^{* * * a, b}$ & $264.8 \pm 15.56^{* * * a, b, c}$ \\
\hline TG (mg /dl) & $131.3 \pm 8.06$ & $136.5 \pm 10.4$ & $175.83 \pm 9^{* * * a, b}$ & $189.6 \pm 14.5^{* * * a, b, c}$ \\
\hline LDL (mg /dl) & $85.75 \pm 13.97$ & $88.58 \pm 11.63$ & $140.83 \pm 11.04^{* * * a, b}$ & $169.16 \pm 13.79^{* * * a, b, c}$ \\
\hline HDL (mg /dl) & $60.25 \pm 3.1$ & $57.25 \pm 3.5$ & $34.25 \pm 4.65^{* * * a, b}$ & $28.58 \pm 3.5^{* * * a, b, c}$ \\
\hline
\end{tabular}

$\mathrm{N}=12$ rats in each group\& data represented as mean and standard deviation ( $\left.\overline{\mathrm{X}}_{ \pm} \mathrm{SD}\right)$.

$\left({ }^{a}\right)=$ significant versus group IA. $\left({ }^{b}\right)=$ significant versus with group IB,$\left(^{c}\right)=$ significant versus group IIA. ${ }^{*} \mathrm{p}<0.05^{* *}=\mathrm{p}<0.01^{* * *}=\mathrm{p}<0.001$

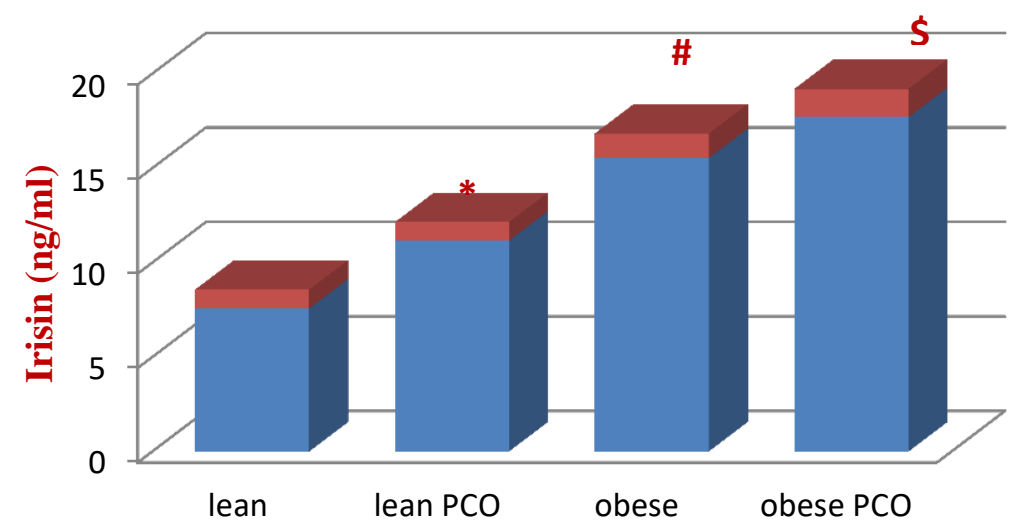

figure (5): Serum levels of irisin in all studied groups

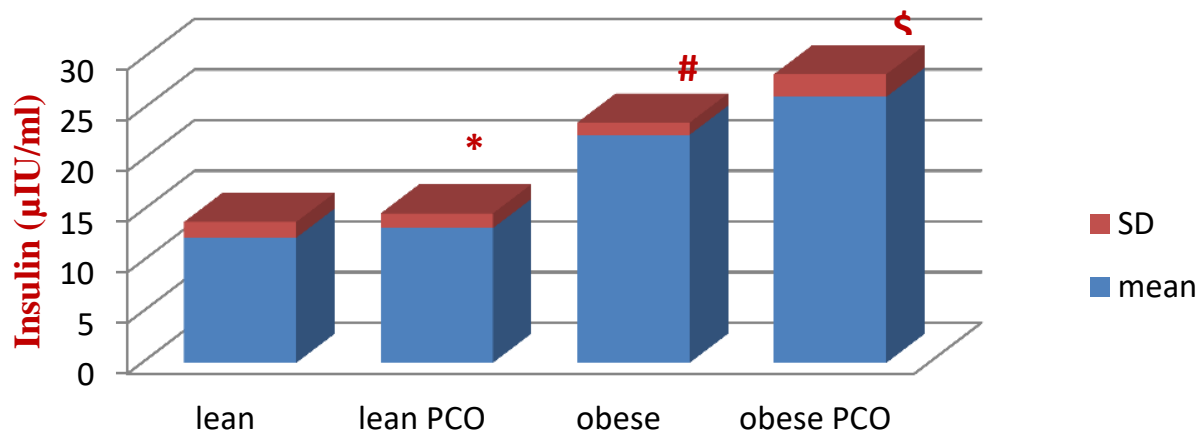

figure (6): Serum levels of Insulin in all studied groups 


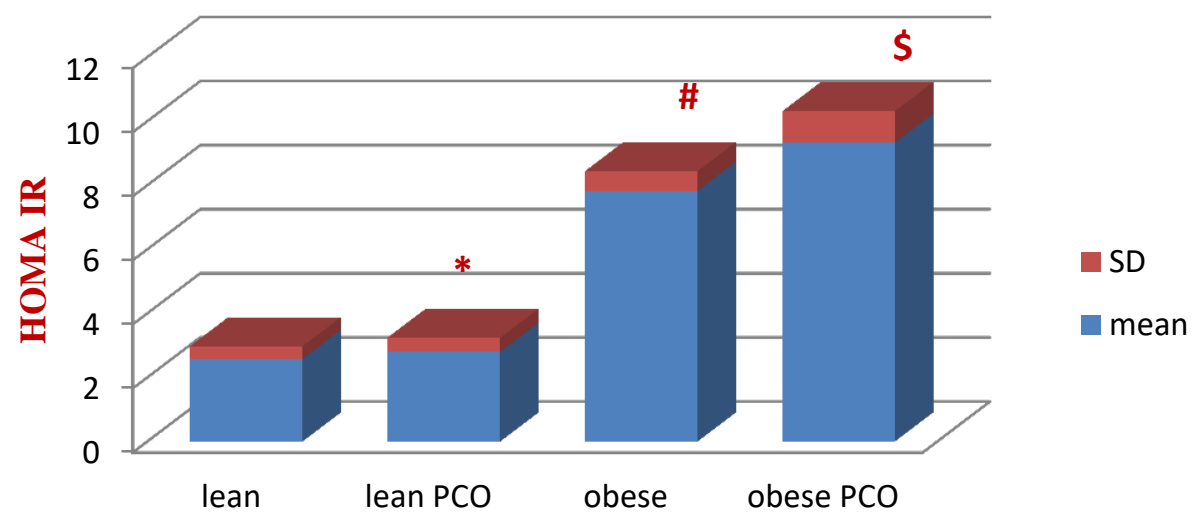

figure (7): HOMA IR in all studied groups

\section{* = group IB VS group IA. $\quad$ " group IIA VS group IA. $\quad{ }^{\$}=$ group IIB VS group IB.}

\section{DISCUSSION}

Irisin is a myokine/adipokine with potential role in obesity and diabetes ${ }^{[25]}$. There are contradictory results about the irisin concentrations in obesity and its relation to both glucose and insulin levels and to insulin resistance $^{[2,3,26]}$.

PCOS is a health problem that affects women of childbearing period and is a common and treatable cause of infertility ${ }^{[27]}$. PCOS women suffer from disturbance in hormonal and metabolic parameters that may affect their health ${ }^{[28,29]}$.

Controversial studies were done about the levels of this myokine in PCOS and its relation to hormonal and metabolic changes of this syndrome ${ }^{[\mathbf{9 , 1 0 , 3 0 ]}}$

This study was carried out to evaluate serum irisin level in letrozole-induced PCOS in both lean and obese female rats, and to find the link between serum irisin level and some metabolic and hormonal changes associated with PCOS.

In the present study, the signs of PCOS induced by letrozole in lean and obese rats was proved by the significant hyperandrogenism (higher serum testosterone level) accompanied by significant reduction in both estradiol and progesterone levels in comparison to control groups, in addition to persistent estrus and histopathological features of cystogenesis ${ }^{[12]}$.

These signs occurred because letrozole blocked cytochrome $\mathrm{P} 450$ aromatase which is responsible for aromatization of testosterone to estradiol [31]. Anovulation was expected because there was a decrease in serum progesterone concentrations ${ }^{[32]}$, increase in the number of atretic and cystic follicles due to disturbed folliculogenesis, moreover a lack of corpus luteam and persistent estrus indicated anovulation ${ }^{[33]}$.

Regarding serum irisin level, there was significant elevation in its level in both lean and obese PCOS groups in comparison with control groups. In addition, obese PCOS group had significant higher levels when compared to lean PCOS group.

This finding is consistent with Cai et al. [34] who showed that circulating irisin level was higher in PCOS patients than in overall healthy controls, and it was also elevated in PCOS patients with higher BMI than those with lower BMI. This suggested that body weight status might be a modulator of circulating irisin alterations in PCOS patients.

In addition, Bostanci et al. ${ }^{[35]}$ found that serum irisin level in PCOS patients was significantly elevated when compared to control group .Li et al. ${ }^{[36]}$ observed a marked increase in circulating irisin levels in obese PCOS women with high free androgen index, in parallel to an evident increase in insulin resistance and hyperandrogenemia.

In the contrary, another study showed that plasma irisin level in PCOS patients did not differ from controls and they found no relation between irisin and other metabolic parameters ${ }^{[10]}$.

Also, Ali et al. ${ }^{[37]}$ reported that serum irisin levels were not significantly differed from their corresponding controls, in both 
obese and non-obese PCOS patients .Whereas, serum irisin levels were elevated significantly in both obese (patients and control) as compared to non-obese (patients and controls),respectively.

Another study has been found that circulating irisin was significantly lower in PCOS patients ${ }^{[38]}$.

In the present study, serum irisin level showed significant positive correlation with BMI.In agreement with this finding, certain studies have reported a positive correlation between serum irisin level and $\mathrm{BMI}^{[39,40]}$, on the other hand Moreno-Navarrete et al. [2] recorded a negative correlation between serum irisin level and BMI. Kurdiova et al. ${ }^{[41]}$ found no correlations between irisin and BMI.

This study showed significant increase in serum $\mathrm{LH}$ and testosterone levels in both lean and obese PCOS groups in comparison with control groups and no significant change in serum FSH. While, there were significant decrease in serum estradiol and progesterone levels in both lean and obese PCOS groups in comparison with control groups.

In addition, this study also showed that the increased level of serum irisin in rats with PCOS showed significant positive correlation with their testosterone levels. This means that the significant hyperandrogenemia observed in PCOS could be considered as a cause responsible for the increased of irisin level.

This may be explained as the increase in lean mass due to higher level of androgen in PCOS ${ }^{[42]}$ could contribute to the secretion of circulating irisin which is secreted from muscle ${ }^{[1]}$.

In addition, Acet et al. ${ }^{[43]}$ found that serum irisin was positively correlated with serum level of total testosterone in PCOS patients but was negatively correlated with HOMA-IR in the overall patient population.

This study showed that serum insulin, glucose levels and HOMA- IR did not show significant change between lean and lean PCOS groups. While there was significant increase in serum insulin, glucose levels and HOMA-IR in both obese and obese PCOS groups in comparison with lean and lean PCOS groups respectively. Moreover, significant elevation in serum insulin level and HOMA- IR in obese PCOS group compared to obese group was reported.

In agreement with these results, Shi et al. ${ }^{[4]}$ reported a rate of abnormal glucose tolerance in PCOS patients. Also, PCOS patients had significantly higher HOMA-IR and fasting blood glucose level compared with those of control women ${ }^{[45]}$.

This can be explained as in case of obesity hyperinsulinemia and IR are accompanied with increased ovarian androgen production ${ }^{[46]}$. Other studies suggest that testosterone may worsen insulin resistance ${ }^{[2,}$ 47].

This study found significant negative correlation between serum irisin level, serum insulin level and HOMA- IR in obese, lean and obese PCOS groups.

The finding in our result is consistent with Acet et al ${ }^{[43]}$ who found that serum irisin level was negatively correlated with HOMAIR in both control and PCOS patients. Furthermore Yan et al. ${ }^{[48]}$ found that in obese adults, there was negative correlation between circulating irisin level and fasting insulin. In addition, a significant negative correlation between serum irisin level and each of fasting glucose level and HOMA-IR was reported in a study carried on healthy children ${ }^{[49]}$.

These data can be approved by the findings of Çath et al. ${ }^{[50]}$ Who found that higher level of irisin may indicate a state of irisin resistance in tissue similar to that of insulin and leptin resistance, or may be interpretated as a compensatory increase in serum irisin level in a trial to increase insulin sensitivity in obese subjects with insulin resistance.

On the other hand, Park et al. [39] reported that, in adults there was positive correlation between serum irisin level and each of fasting glucose level and HOMA-IR. Moreover, in both PCOS and normal women, circulating irisin level was positively correlated with BW, BMI, HOMA- IR ${ }^{[9]}$. While, Bluher et al. ${ }^{[51]}$ found no relation between serum irisin level and other metabolic parameters associated with obesity (e.g., glucose and insulin) in obese children.

There is a new prove that exogenous administration of recombinant irisin in animal models or in vitro irisin treatment in cell 
culture systems is associated with enhanced glycemic control and improved insulin resistance $^{[\mathbf{5 2}, \mathbf{5 3}]}$.

This study showed that total serum cholesterol, TG, LDL and HDL levels did not show significant change between lean and lean PCOS group. While there was significant increase in total serum cholesterol, TG, LDL and decrease in HDL levels in both obese and obese PCOS groups in comparison with lean and lean PCOS groups respectively. Furthermore, there was significant increase in total cholesterol, TG, LDL and decrease in HDL levels in obese PCOS group when compared to obese group.

In addition, there was significant negative correlation between serum irisin level and total serum cholesterol, TG and LDL levels. On the other hand, there was significant positive correlation between serum irisin level and serum HDL levels in obese and both lean \& obese PCOS groups.

These results are in agreement with Tasali et al ${ }^{[54]}$ who found that PCOS women have a high risk of disturbed lipid metabolism disorder.

Conflicting results have been reported between serum irisin level and lipid profile. A positive correlation between serum irisin level and TG, TC, and LDL has been recorded by Park et al. ${ }^{[39]}$ and on the other hand MorenoNavarrete et al. ${ }^{[2]}$ and Yan et al. ${ }^{[48]}$ found that there was no correlation between irisin and lipid parameters.

Reinehr et al. ${ }^{[55]}$ observed that serum irisin level was positively correlated with LDL-C and TG levels, and negatively correlated with HDL-C in childhood.

While Al-Daghri et al. ${ }^{[49]}$ reported that serum irisin level was positively correlated with HDL level in normal-weight children. Other studies showed that serum irisin level w7] negatively correlated with HDL level ${ }^{[56,}$

Irisin may perform its effects on lipid synthesis, particularly HDL-C, but low HDL$\mathrm{C}$ level may produce a direct feedback effect on myocytes, enhancing the release of irisin to restore the altered metabolism ${ }^{[\mathbf{5 7}, \mathbf{5 8}]}$.

Discrepencies in these results and other may be related to difference in species, irisin kits, study design, and duration of the experiment.

In conclusion: High serum irisin level in PCOS and obese groups were negatively correlated with insulin resistance and hyperlipidemia, while positively correlated with BMI and hyperandrogenemia. Irisin may have a role in the development of polycystic ovary syndrome. Moreover, the finding of high serum irisin level in PCOS may provide a novel biomarker for detection of this syndrome.

\section{RECOMMENDATION}

Further studies are needed to clarify the exact role of exogenous administration of irisin in hormonal changes in infertility.

Acknowledgment to Professor Kamal Eleshishi, pathology department, faculty of Medicine, Zagazig University for performing the histological study

\section{REFERENCES}

1- Bostrom P, Wu J, Jedrychowski MP, Korde A, Ye L, Lo JC, et al: A PGC1-[agr]dependent myokine that drives brown-fat-like development of white fat and thermogenesis. Nature 2012; 481(7382):463-468.

2- Moreno-Navarrete JM, Ortega F, Serrano M, Guerra E, Pardo G, Tinahones F, et al: Irisin is expressed and produced by human muscle and adipose tissue in association with obesity and insulin resistance. J Clin Endocrinol Metab, 2013; 98(4):769-778.

3- Meili Y, Peihong C, Hua J, Xinmiao X, Gao T, Yang L, et al: Circulating levels of irisin in middle-aged first-degree relatives of type 2 diabetes mellitus - correlation with pancreatic $\beta$-cell function. Diabetology \& Metabolic Syndrome, 2014; 6:133

4- Hee PK, Zaichenko $L$, Brinkoetter $M$, Thakkar B, Sahin-Efe A and Joung KE: Circulating irisin in relation to insulin resistance and the metabolic syndrome. $\mathrm{J}$ Clin Endocrinol Metab. 2013; 98 (12):4899-907.

5- Badawy A and Elnashar A: Treatment options for polycystic ovary syndrome. International Journal of Women's Health 2011; 3: 25-35.

6- Cardozo E, Pavone ME and HirshfeldCytron JE: Metabolic syndrome and oocyte quality. Trends Endocrinol Metab. 2011; 22:103-9.

7-Kocer D1, Bayram F and Diri $\mathbf{H}$ : The effects of metformin on endothelial dysfunction, 
lipid metabolism and oxidative stress in women with polycystic ovary syndrome. Gynecol Endocrinol. 2014; 30(5):367-71

8-Manco M, Castagneto-Gissey L, Arrighi E, Carnicelli A, Brufani C, Luciano R, et al : Insulin Dynamics in Young Women with Polycystic Ovary Syndrome and Normal Glucose Tolerance across Categories of Body Mass Index. PLoS ONE, 2014; 9(4): e92995.

9-Li M, Yang M, Zhou X, Fang X, Hu W, Zhu $\mathbf{W}$, et al: Elevated circulating levels of irisin and the effect of metformin treatment in women with polycystic ovary syndrome. J Clin Endocrinol Metab. 2015; 100(4):1485-93

10- Pukajlo K, Laczmanski L, Kolackov K, Kuliczkowska-Plaksej J, Bolanowski M., Milewicz A, et al: Irisin plasma concentration in pcos and healthy subjects is related to body fat content and android fat distribution.Gynecol.Endocrinol.2015; 31, 907-911.

11- Cha MC, Chou CJ and Boozer CN: Highfat diet feeding reduces the diurnal variation of plasma leptin concentration in rats. Metabolism.2000; 49(4):503-7.

12-Kafali H, Iriadam M, Ozardali I and Demir $\mathbf{N}$ : Letrozole-induced polycystic ovaries in the rat: a new model for cystic ovarian disease. Arch Med Res.2004; 35:103-8.

13- Marcondes FK, Bianchi FJ and Tanno AP: Determination of the estrous cycle phases of rats: some helpful considerations. Braz. J. Biol.2002; 62(4A):609-614.

14- Goldman JM, Murr AS and Cooper RL: The rodent estrous cycle characterisation of vaginal cytology and its utility in toxicological studies. Birth Defects Research (Part B), 2007; 80: 84-97.

15- Novelli E, Diniz Y, Galhardi C, Ebaid G, Rodrigues $\mathrm{H}$, Mani $\mathrm{F}$, et al: Anthropometrical parameters and markers of obesity in rats Laboratory Animals Ltd. Laboratory Animals 2007; 41, 111-119.

16- Nishizawa H, Shimomura I and Kishida K: Androgens decrease plasma adiponectin, an insulin-sensitizing adipocyte-derived protein. Diabetes, 2002; 51: 2734-2741.

17- Tietz N W: Clinical Guide to Laboratory Tests,W.B. Saunders, Co., Philadelphia, 1995; 509-512,

18- Temple R C, Clark $\mathbf{P} \mathbf{M}$ and Hales $\mathbf{C}$ N: Measurement of insulin secretion in type 2 diabetes: problems and pitfalls. Diabetic Medicine, 1992; 9: 503-512.
19- Sun G, Bishop J, Khalili S, Vasdev S, Gill V, Pace, et al: Serum visfatin concentrations are positively correlated with serum triacylglycerols and downregulated by overfeeding in healthy young men. Am J Clin Nutr, 2007; 85(2): 399-404.

20- Fossati, P.: Principle Lab. Clin Chem, 1982; 28: 2077-20779.

21- Nauck M, März W, Jarausch J, Cobbaert C, Sägers A, Bernard D, et al: Multicenter evaluation of a homogeneous assay for HDL-cholesterol without sample pretreatment. Clin Chem, 1997; 43(9):1622-1629.

22- Friedwald W T, Levy R I and Fredrickson D S: Estimation of the concentration of low-density lipoprotein cholesterol in plasma, without use of the preparative ultracentrifuge. Clin Chem, 1972; 18(6): 499-502.

23- Baravalle C, Salvetti NR, Mira GA, Lorente JA and Ortega HH: The role of ACTH in the pathogenesis of polycystic ovarian syndrome in rats: hormonal profiles and ovarian morphology. Physiol Res. 2007; 56(1):67-78.

24-Kirkwood B R: Essentials of medical statistics. Blackwell scientific publication, Oxford, London, 1989; pp 151.

25- Belviranli M, Okudan N and Çelik F : Association of circulating irisin with insulin resistance and oxidative stress in obese women. Hormone and Metabolic Research, 2016; 48(10), 653-657.

26- Liu J J, Wong M D, Toy W C, Tan C S, Liu $\mathrm{S}, \mathrm{Ng} \mathrm{X} \mathrm{W}$, et al: Lower circulating irisin is associated with type 2 diabetes mellitus. $\mathbf{J}$ Diabetes Complications, 2013; 27(4):365369.

27- McCartney C R and Marshall J C : Polycystic ovary syndrome. New England Journal of Medicine, 2016; 375(1), 54-64.

28- Kocer D1, Bayram $\mathbf{F}$ and Diri H: The effects of metformin on endothelial dysfunction, lipid metabolism and oxidative stress in women with polycystic ovary syndrome. Gynecol Endocrinol. 2014; 30(5):367-71

29- Manco M, Castagneto-Gissey L, Arrighi E, Carnicelli A, Brufani C, Luciano R, et al: Insulin Dynamics in Young Women with Polycystic Ovary Syndrome and Normal Glucose Tolerance across Categories of Body Mass Index. PLoS ONE 2014; 9(4): e92995 
30- Wang C, Zhang X Y, Sun Y, Hou X G and Chen L. Higher circulating irisin levels in patients with polycystic ovary syndrome: a meta-analysis. Gynecological Endocrinology, 2018; 34(4), 290-293.

31- Van Voorhis BJ, Dunn MS, Snyder GD and Weiner CP: Nitric oxide: an autocrine regulator of human granulosa-luteal cell steroidogenesis. Endocrinology, 1994; 135:1799-1806.

32- Meenakumari KJ, Agarwal S, Krishna A and Pandey LK: Effects of metformin treatment on luteal phase progesterone concentration in polycystic ovary syndrome. Braz J Med Biol Res. 2004; 37 : 1637-44.

33- Rezvanfara M A, Rezvanfara MA, Ahmadic A, Shojaei-Saadid H A Baeeria $M$ and Abdollahia M: Molecular mechanisms of a novel selenium-based complementary medicine which confers protection against hyperandrogenism induced polycystic ovary. Theriogenology, 2012; 78: 620-631.

34- Cai Xue, Qiu Shanhu, Li Ling, Zügel Martina, Steinacker JM and Schumann Uwe : Circulating irisin in patients with polycystic ovary syndrome: a metaanalysis. Reproductive Biomedicine online, 2018; (36): $172-180$.

35- Bostanci M S, Akdemir N, Cinemre B, Cevrioglu A S, Ozden S and Unal O: Serum irisin levels in patients with polycystic ovary syndrome. Eur. Rev. Med. Pharmacol. Sci. 2015; 19: 4462-4468.

36- Li H, Xu X, Wang X., Liao X, Li L, Yang G, et al: Free androgen index and irisin in polycystic ovary syndrome. J. Endocrinol. Invest. 2016; 39: 549-556.

37- Ali S H, Al-Nuaimi A L I M A and AlMusawi B J : Serum irisin and leptin levels in obese and non-obese women with polycystic ovary syndrome with reference to glucose homeostasis. Int J Pharm Pharm Sci, 2016; 8 (10): 276-283.

38- Abali R, Temel Yuksel I, Yuksel M A, Bulut B, Imamoglu M, Emirdar V, et al: Implications of circulating irisin and Fabp4 levels in patients with polycystic ovary syndrome. J. Obstet. Gynaecol.2016; 36: 897-901.

39- Park K H, Zaichenko L, Brinkoetter M, Thakkar B, Sahin-Efe A, Joung K.E, et al: Circulating irisin in relation to insulin resistance and the metabolic syndrome. $\mathbf{J}$ Clin Endocrinol Metab. 2013; 98(12): 4899-48907.
40- Crujeiras AB, Pardo M, Arturo RR, NavasCarretero S, Zulet MA, Martinez JA, et al: Longitudinal variation of circulating irisin after an energy restriction induced weight loss and following weight regain in obese men and women. Am J Hum Biol. 2014; 26(2):198-207.

41- Kurdiova T, Balaz M, Vician M, Maderova D, Vlcek M, Valkovic L, et al: Effects of obesity, diabetes and exercise on Fndc5 gene expression and irisin release in human skeletal muscle and adipose tissue: in vivo and in vitro studies. J Physiol, 2014; 592(5):1091-107.

42- Notelovitz M. Androgen effects on bone and muscle. Fertil Steril 2002; 77: S34-S41.

43- Acet M, Celik N, Acet T, Ilhan S, Yardim M, Aktun $\mathbf{H}$ L, et al: Serum and follicular fluid irisin levels in poor and high responder women undergoing IVF/ICSI. Eur Rev Med Pharmacol Sci, 2016; 20(10), 1940-1946.

44- Shi Y H, Zhao D N, Zhao J L, You L, Liu H, Sun M, et al: Characteristics of glucose metabolism in non-obese and obese women with polycystic ovarian syndrome. Chinese Obstet Gynecol. 2010; 45: 575-577.

45- Gao S, Cheng Y, Zhao L, Chen Y and Liu Y: The relationships of irisin with bone mineral density and body composition in Pcos patients. Diabetes Metab. Res. Rev. 2016; 32, 421-428.

46- Qu J, Wang Y, Wu X, Gao L, Hou L and Erkkola R: Insulin resistance directly contributes to androgenic potential within ovarian theca cells. Fertil Steril. 2009; 91:1990-1997.

47- Patel SM, Ratcliffe SJ, Reilly MP, Weinstein R, Bhasin S, Blackman MR, et al: Higher serum testosterone concentration in older women is associated with insulin resistance, metabolic syndrome, and cardiovascular disease. J Clin Endocrinol Metab. 2009; 94:4776-4784.

48- Yan B, Shi X, Zhang H, Pan L, Ma Z, Liu S, et al: Association of Serum Irisin with Metabolic Syndrome in Obese Chinese Adults. PLOS ONE, 2014; 9(4): e94235.

49- Al-Daghri NM, Alkharfy KM, Rahman S, Amer OE, Vinodson B, Sabico S, et al: Irisin as a predictor of glucose metabolism in children: Sexually dimorphic effects. European Journal of Clinical Investigation, 2014; 44(22):119-124.

50- Çatlı G, Küme T, Tuhan H Ü, Anık A, Çalan Ö G, Böber E, et al: Relation of serum irisin level with metabolic and 
antropometric parameters in obese children. Journal of diabetes and its complications, 2016; 30(8), 1560-1565.

51- Bluher S, Panagiotou G, Petroff D, Markert J, Wagner A, Klemm T, et al: Effects of a 1-year exercise and lifestyle intervention on irisin, adipokines, and inflammatory markers in obese children. Obesity (Silver Spring), 2014; 22(7), 17011708.

52- Jeong Lee H, Ok Lee J, Kim N, Kwan Kim J, Ip Kim H, Woo Lee Y, et al: Irisin, a novel myokine, regulates glucose uptake in skeletal muscle cells via ampk. Mol Endocrinol 2015; 29(6): 873-81.

53- Zhang Y, Li R, Meng Y, Li S, Donelan W, Zhao Y, et al: Irisin stimulates browning of white adipocytes through mitogen-activated protein kinase p38 map kinase and erk map kinase signaling. Diabetes 2014; 63(2):51425.

54- Tasali E, Van Cauter E and Ehrmann DA: Relationships between sleep disordered breathing and glucose metabolism in polycystic ovary syndrome. J Clin Endocrinol Metab 2006; 91: 36-42.
55- Reinehr T, Elfers C, Lass N and Roth CL: Irisin and its relation to insulin resistance and puberty in obese children: A longitudinal analysis. The Journal of Clinical Endocrinology and Metabolism, 2015; 100: 2123-2130.

56- Huh JY, Panagiotou G, Mougios V, Brinkoetter M, Vamvini MT, Schneider BE, et al: FNDC5and irisin in humans: I. Predictors of circulating concentrations in serum and plasma and II. mRNA expression and circulating concentrations in response to weight loss and exercise. Metabolism 2012; 61: 17251738.

57- Panagiotou G, Mu L, Na B, Mukamal KJ and Mantzoros CS.: Circulating irisin, omentin-1, and lipoprotein subparticles in adults at higher cardiovascular risk. Metabolism, 2014; 63: 1265-1271.

58- Lehti M, Donelan E, Abplanalp W, AlMassadi O, Habegger KM, Weber J, et al: High-density lipoprotein maintains skeletal muscle function by modulating cellular respiration in mice. Circulation, 2013; 128, $2364-2371$. 